\section{Conjecture on the Visual Estimation of Relative Radial Motion}

THE ability to estimate accurately the time interval until the juncture of an observer with an object moving with relative radial velocity towards the observer seems to be highly developed in humans and many animals. In many practical situations, of probable importance for survival, it is not the distance of the object from the observer which is of interest, nor is it the velocity of the object, but rather the ratio of the two, which gives the time taken by the object to reach the observer. The importance of this parameter has certainly been rencwed in the age of the motor car. The popularity of ball games may be partly due to the exercise of this faculty otherwise dormant in a sedentary era.

I wish to show here that there is sufficient information available on the monocular retina to allow the observer to estimate the time of juncture with the moving object without the need for absolute knowledge of the distance of the object, its size or its velocity.

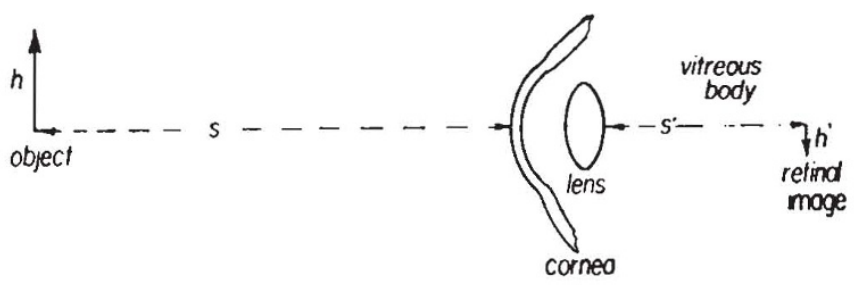

Fig. 1 Optical system of the eye of the observer and an object moving with uniform radial velocity towards the observer (not to scale).

Fig. 1 shows schematically an optical system representing the eye of the observer and an object of size $h$ at a distance $S$ from the observer. If $v$ is the relative uniform velocity of the object towards the observer then the time interval until juncture is given by

$$
t=\begin{aligned}
& S \\
& v
\end{aligned}
$$

For paraxial rays Lagrange's law gives for the image size $h^{\prime}$ on the retina

$$
h^{\prime}=\begin{array}{ll}
h & S^{\prime} \\
S & n^{\prime}
\end{array}
$$

where $S^{\prime}$ is the distance of the retina from the lens and $n^{\prime}$ the index of refraction of the vitreous humour.

Differentiating equation (2) with respect to time gives

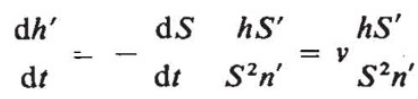

because $S^{\prime}$ may be assumed constant.

Dividing equation (2) by equation (3) gives

$$
\frac{h^{\prime}}{\frac{\mathrm{d} h^{\prime}}{\mathrm{d} t}}=\frac{S}{v}=t
$$

that is, the time interval of interest.

Thus a knowledge of the instantaneous image size and its time derivative is sufficient to determine, with monocular vision alone, the juncture interval without requiring absolute knowledge of $S, v$ or even $h$.

The verification of the conjecture that the visual system is sufficiently adept to estimate the time interval to juncture from the limited but sufficient retinal information is an interesting experimental problem.

Commenting on this conjecture, Professor R. L. Gregory (private communication) has remarked: "Visual size is not, of course, merely a matter of retinal size and it is not very clear how the information of the retinal size is retained after 'constancy scaling'. This itself is an interesting question and I would like to know the answer."

\title{
Hershel Weinberger
}

\section{National Physical Laboratory of Israel, Jerusalem}

Received October 13, 1970.

\section{Changes in Adrenal Enzymes synthesizing Catecholamines in Attack Behaviour evoked by Hypothalamic Stimulation in the Cat}

IN the cat, electrical stimulation ${ }^{1.2}$ or selective lesions of the brain $^{3}$ can evoke an affected display of anger, called sham rage ${ }^{3}$. This emotional display is often coupled with directed prey killing or attack behaviour ${ }^{2}$. Associated with this is a widespread activation of the sympathetic nervous system and a release of the catecholamines, noradrenaline and adrenaline, from the adrenal gland ${ }^{4,5}$. It was recently demonstrated that, when prolonged, increased neural release of adrenal catecholamines is associated with increased activity of tyrosine hydroxylase ${ }^{6,7}$, the enzyme of the rate-limiting step in catecholamine biosynthesis $^{8}$. The increase in enzyme activity is presumably the result of production of new enzyme ${ }^{6.9}$. We have sought to determine whether the induction of attack behaviour in the cat by electrical stimulation of the hypothalamus, if sustained over several days, can lead to similar changes in adrenal tyrosine hydroxylase and also the adrenal enzyme phenylethenol amine-N-methyl transferase (PNMT) which converts norepinephrine to epinephrine.

In a preliminary operation, adult cats were placed under halothane anaesthesia and electrode guides were stereotactically placed through drill holes in the calvarium, over sites in the posterior hypothalamus from which attack behaviour can be evoked $^{2}$. Several days later the animals were placed in an observation cage with an anaesthetized rat and an insulated monopolar electrode with a $0.5 \mathrm{~mm}$ bare tip was lowered and fixed into the locus at which rat-killing behaviour could be evoked by electrical stimulation. The anode was a screw embedded in the skull. In controls, the electrode was placed in immediately adjacent hypothalamic regions from which some motor activity but not rage or prey-killing could be elicited. Animals were electrically stimulated from a constant current stimulator for $90 \mathrm{~min}$ in the morning and in the afternoon for 3 consecutive days. The stimulus programme consisted of 2 min trains of square wave pulses $(0.5 \mathrm{~ms}$ duration, $0.5-2.0 \mathrm{~mA}$, 70 c.p.s.) each followed by a 3 min rest. After the last stimulation session, the animals were lightly anaesthetized with

Table 1 Enzyme Activity in Adrenal Gland after Brain Stimulation Tyrosine hydroxylase * ${ }^{*}$ PNMT *

Control

(n)

3 day stimulation, no attack

(n)

3 day stimulation, attack (n)

* Activity expressed by nmol of product $/ 100 \mathrm{mg} / 20 \mathrm{~min} \pm$ s.e.m. $\dagger$ Difference from control significant $(P<0.001)$.

NS, Difference from control not significant $(P>0.05)$. 